\title{
LINEAR TRANSFORMATIONS WITH CHARACTERISTIC SUBSPACES THAT ARE NOT HYPERINVARIANT
}

\author{
Pudji Astuti And Harald K. WimMer
}

\begin{abstract}
If $f$ is an endomorphism of a finite dimensional vector space over a field $K$ then an invariant subspace $X \subseteq V$ is called hyperinvariant (respectively, characteristic) if $X$ is invariant under all endomorphisms (respectively, automorphisms) that commute with $f$. According to Shoda (Math. Zeit. 31, 611-624, 1930) only if $|K|=2$ then there exist endomorphisms $f$ with invariant subspaces that are characteristic but not hyperinvariant. In this paper we obtain a description of the set of all characteristic non-hyperinvariant subspaces for nilpotent maps $f$ with exactly two unrepeated elementary divisors.
\end{abstract}

Mathematics subject classification (2010): 15A18, 47A15, 15A57.

Keywords and phrases: hyperinvariant subspaces, characteristic subspaces, invariant subspaces, Ulm invariants, characteristic hull, exponent, height.

\section{REFERENCES}

[1] P. Astuti And H. K. Wimmer, Hyperinvariant, characteristic and marked subspaces, Oper. Matrices 3 (2009), 261-270.

[2] P. Astuti And H. K. Wimmer, Characteristic and hyperinvariant subspaces over the field GF(2), Linear Algebra Appl. 438 (2013), 1551-1563.

[3] R. BAER, Types of elements and characteristic subgroups of Abelian groups, Proc. London Math. Soc. 39 (1935), 481-514.

[4] KH. BenABDAllah AND B. ChaRles, Orbits of invariant subspaces of algebraic linear operators, Linear Algebra Appl. 225 (1995), 13-22.

[5] R. BRU, L. RODMAN, AND H. SCHNEIDER, Extensions of Jordan bases for invariant subspaces of a matrix, Linear Algebra Appl. 150 (1991), 209-226.

[6] P. A. Fillmore, D. A. Herrero, AND W. E. LongstafF, The hyperinvariant subspace lattice of a linear transformation, Linear Algebra Appl. 17 (1977), 125-132.

[7] L. Fuchs, Infinite Abelian Groups, Vol. I., Academic Press, New York, 1973.

[8] L. Fuchs, Infinite Abelian Groups, Vol. II., Academic Press, New York, 1973.

[9] I. Gohberg, P. LANCASTER, AND L. Rodman, Invariant Subspaces of Matrices with Applications, Wiley, New York, 1986.

[10] I. KaPLANSKy, Infinite Abelian Groups, University of Michigan Press, Ann Arbor, 1954.

[11] W. E. LONGSTAFF, A lattice-theoretic description of the lattice of hyperinvariant subspaces of a linear transformation, Can. J. Math. 28 (1976), 1062-1066.

[12] W. E. LONGSTAFF, Picturing the lattice of invariant subspaces of a nilpotent complex matrix, Linear Algebra Appl. 56 (1984), 161-168.

[13] K. SHODA, Über die characteristischen Untergruppen einer endlichen Abelschen Gruppe, Math. Zeit. 31 (1930), 611-624.

[14] D. A. Suprunenko And R. I. Tyshrevich, Commutative Matrices, Academic Press, New York, 1968. 